\title{
The Time-Dependent Schrödinger Equation: The Need for the Hamiltonian to be Self-Adjoint
}

\author{
Vanilse S. Araujo, \\ Escola de Engenharia Maua, São Paulo, Brazil and Faculdade de Engenharia da Fundação Santo Andre, São Paulo, Brazil \\ F. A. B. Coutinho, \\ Faculdade de Medicina da Universidade de São Paulo, São Paulo, 01246-903, Brazil \\ F. M. Toyama \\ Department of Information and Communication Sciences, Kyoto Sangyo University, Kyoto 603-85555 Japan
}

Received on 20 December, 2007

\begin{abstract}
We present some simple arguments to show that quantum mechanics operators are required to be self-adjoint. We emphasize that the very definition of a self-adjoint operator includes the prescription of a certain domain of the operator. We then use these concepts to revisit the solutions of the time-dependent Schroedinger equation of some well-known simple problems - the infinite square well, the finite square well, and the harmonic oscillator. We show that these elementary illustrations can be enriched by using more general boundary conditions, which are still compatible with self-adjointness. In particular, we show that a puzzling problem associated with the Hydrogen atom in one dimension can be clarified by applying the correct requirements of self-adjointness. We then come to Stone's theorem, which is the main topic of this paper, and which is shown to relate the usual definitions of a self-adjoint operator to the possibility of constructing well-defined solutions of the timedependent Schrödinger equation.
\end{abstract}

Keywords: Operator domains; Self-adjointness; Stone theorem; Quantum Mechanics; Operator exponential

\section{INTRODUCTION}

In quantum mechanics observables are represented by operators acting on the functions belonging to the Hilbert space of the system under consideration [1]. However, in contrast to the mathematical literature, where operators are defined by their action (that is, what they do to the functions on which they operate) and by their domain (that is, the set of functions on which they operate), in the physical literature domains are almost never mentioned and operators are defined only by their actions. Operators in infinite dimensional Hilbert space are not defined for all the functions of the space, and this suggests that one should be aware of situations where domains are important.

The reason why the domains of the operators are so important, even in physics, is that we need the operators in quantum mechanics to be self-adjoint and operators are self-adjoint only in well-defined and prescribed domains. But why is it so important for operators in quantum mechanics to be selfadjoint? There are two important reasons: The first one is that the eigenvalues are real and the eigenfunctions form a complete set of orthogonal "functions" so that any function of the Hilbert space of the system in consideration can be expanded in this set. (The reason for the quotation marks in the word functions will become clear later.) The second reason is that only if the Hamiltonian $H$ of the system is a self-adjoint operator, the time-dependent Schrödinger equation $i \hbar \frac{\partial}{\partial t} \varphi=H \varphi$ has a unique solution-such that $\|\varphi(t)\|^{2}=\|\varphi(0)\|^{2}-$ for all times. This is the content of Stone's theorem [2] that we will explain in detail in section 3 , and has to do with the possibility of constructing the exponential of an operator [3], in this case the operator $\exp \left[-\frac{i}{\hbar} H t\right]$. However, it is hard to see how those two properties are linked with domains. The objective of this paper is to make this link intuitively clear

Until quite recently, domains or self-adjointness were not mentioned in the physical literature. However, in the last few years or so, some articles [4], [5], [6], [7] in the physics pedagogical literature begun to point out examples where domains of operators are essential to the full solution of the problems posed. We are aware of only three pedagogical articles published before those articles [6-9] that mention domains and self-adjointness. One is by Jordan [8], another by Capri [9], and finally an article by Zhu and Klauder [10] that relates lack of self -adjointness with strange classical behavior.

It is interesting to try to understand why it is possible to neglect domains in the physical literature. First, it is true that domains rarely cause problems. This is indeed so, but Gieres [11] cites seven examples of physical puzzles caused by the manipulation of operators neglecting attention to their domains. Second, if domains are not mentioned, it is natural to think that domains are automatically specified. One tends to think that if $\widehat{A}$ is an operator and $\widehat{A} \varphi$ belongs to the Hilbert space of the system under consideration then $\varphi$ belongs to the domain of the operator. It is true for that for $\varphi$ to belong to the domain of an operator $\widehat{A}, \widehat{A} \varphi$ must belong to the Hilbert space of the system and we will assume this in the remaining of the paper without further comment. However, although there are operators with good properties in such a large domain (see Appendix 1 and the example (1.1) and (1.3) below) in general it is necessary to restrict the domain by specifying boundary conditions to be obeyed by the functions of the domain. The domains are specified in such a way, but it is hard to see that this has been done because this fact is never mentioned. As we will see domains are specified by the boundary conditions imposed when solving the time-independent Schrödinger equation. 
This paper is organized as follows. In the next section we recapitulate briefly, and in a non- technical way, what a selfadjoint operator is, and apply self-adjointness to the solution of the time-independent Schrödinger equation. The examples chosen to illustrate the concept of self- adjointness are all the familiar examples found in quantum mechanics textbooks: the infinite square well, the harmonic oscillator, the hydrogen atom, etc. However, it is shown how the concept of self-adjoint extension enriches these familiar illustrations, and clarifies also a puzzling situation (the hydrogen atom in one dimension). Next, in section 3, we state Stone's theorem and in section 4 we show how this theorem is related to the possibility of constructing the exponential of an operator emphasizing how this construction is linked to the domain of the operator. This completes our main task in this paper that is to show why self-adjointness is essential to operators in quantum mechanics. In the Appendix, for completeness, we present two examples-the harmonic oscillator and the finite square wellof Hamiltonians that do not require boundary conditions for the definition of their domains. Finally, in Appendix 2, we deduce an unusual boundary condition at $r=0$ for the s-wave radial part of the Hamiltonian describing the hydrogen atom.

\section{SELF-ADJOINTNESS AND THE TIME-INDEPENDENT SCHRÖDINGER EQUATION}

In this paper we will confine ourselves, for definiteness, to one-dimensional systems. Assume a particle that is free to move in the entire real line. Then, the Hilbert space of this system is the set of functions such that

$$
\int_{-\infty}^{+\infty} \varphi^{*}(x) \varphi(x) d x=\int_{-\infty}^{+\infty}|\varphi(x)|^{2} d x=\|\varphi\|^{2}=\text { finite },
$$

where $\varphi^{*}(x)$ denotes the complex conjugate of $\varphi(x)$. The inner product between two function $\varphi_{1}(x)$ and $\varphi_{2}(x)$ in this space is defined by

$$
<\varphi_{1} \mid \varphi_{2}>=\int_{-\infty}^{+\infty} \varphi_{1}^{*}(x) \varphi_{2}(x) d x
$$

Given a certain operator $\widehat{O}$ we define its Hermitian conjugate as the operator $\widehat{O}^{\dagger}$ defined by

$$
\begin{gathered}
<\varphi_{1} \mid\left(\widehat{O} \varphi_{2}\right)>=\int_{-\infty}^{+\infty} \varphi_{1}^{*}(x)\left(\widehat{O} \varphi_{2}(x)\right) d x=\int_{-\infty}^{+\infty}\left(\widehat{O}^{\dagger} \varphi_{1}(x)\right)^{*} \varphi_{2}(x) d x \\
=<\left(\widehat{O}^{\dagger} \varphi_{1}\right) \mid \varphi_{2}>.
\end{gathered}
$$

When the operators $\widehat{O}^{\dagger}$ and $\widehat{O}$ have the same action (that is they do the same thing to the functions on which they operate) and equation (3) holds, they are called Hermitian (symmetric by mathematicians). If in addition they have the same domain then they are self-adjoint. The distinction between Hermitian operators and self-adjoint operators is rather subtle. To clarify the distinction reference [5] presents two examples of operators with the same action but with different domains so that in the first example the operator is Hermitian and in the second the operator is self-adjoint. (One should note that the distinction between Hermitian and self-adjoint operators disappears for bounded operators-which are defined for all the functions of the Hilbert space- and hence for operators in finite dimensional spaces, that is for matrices.)

As mentioned in the introduction, when solving the timeindependent Schrö-dinger equation, it is in general necessary to restrict the domains of operators by imposing boundary conditions so that they become self-adjoint. In the remaining of this section we present examples of this procedure. The examples (1.1) and (1.3) are illustrations of operators where the domains are restricted only by their action. The other examples are illustrations of cases where boundary conditions are necessary in addition to the restrictions imposed by the action.
Example 1.1) The operator $\hat{x}$

In this example we consider the operator $\hat{x}$, that is multiplication by $x$, acting on the Hilbert space of the functions given by equation (1). A "natural" domain for this operator is the set of functions $\varphi(x)$ that in addition to having finite norm also obeys

$$
\int_{-\infty}^{+\infty}|x \varphi(x)|^{2} d x=\text { finite }
$$

Is this operator so defined self-adjoint? Consider two functions $\varphi_{1}(x)$ and $\varphi_{2}(x)$ obeying equation (4). We have that

$$
\int_{-\infty}^{+\infty} \varphi_{1}^{*}(x)\left(x \varphi_{2}(x)\right) d x=\int_{-\infty}^{+\infty}\left(x \varphi_{1}(x)\right)^{*} \varphi_{2}(x) d x,
$$

and this happens if both $\varphi_{1}(x)$ and $\varphi_{2}(x)$ obey equation (4). So the operator $\hat{x}$ is self- adjoint in this "natural" domain.

Example 1.2) A criterion of self-adjointness

It is generally difficult to check domains. So we present below a result that helps to recognize if an operator is selfadjoint in its domain. This result is explained in reference 
[5], and is a basic criterion of self-adjointness. To see if an operator $\widehat{O}$ is self-adjoint consider the equations

$$
\widehat{O}^{\dagger} \varphi(x)= \pm i \eta \varphi(x)
$$

where $\eta$ is a constant which maintains the dimensional consistence of the equations. If the above equations have no square integrable solutions in the proposed domain then the operator is self-adjoint. Clearly the equations

$$
\hat{x}^{\dagger} \varphi(x)=x \varphi(x)= \pm i x_{0} \varphi(x)
$$

have no solution, except $\varphi(x) \equiv 0$, in the entire Hilbert space. (The Dirac delta function is not admissible because it is not square integrable [12].) So the operator $\hat{x}$ is self-adjoint in its "natural"domain as shown in the previous example by inspection.

\section{Example 1.3) The operator momentum}

The operator momentum, $\hat{p}=\frac{\hbar}{i} \frac{d}{d x}$, acting on the space of functions given by equations (1) is self-adjoint in its "natural" domain, that is, the domain are functions $\varphi(x)$ such that $\hat{p} \varphi(x)$ are square integrable (satisfies equation (1)). This can be easily deduced from the fact that equation (6) with $\widehat{O}$ replaced by $\hat{p}$ has no square integrable solutions in this space.

The next two examples (examples 1.4 and 1.5) show how the specification of the domains by imposing boundary conditions enriches even the most common examples found in quantum mechanics textbooks. (Many other examples-which however are more advanced- of how to modify simple problems by specifying boundary conditions and their physical significance can be found in reference [5].)
Example 1.4) A free particle confined to the right half of the real axis with the Hamiltonian defined in a domain where it is self-adjoint.

Consider the operator whose action is proportional to minus the second derivative, that is, $\widehat{O}=-\frac{\hbar^{2}}{2 m} \frac{d^{2}}{d x^{2}}$, defined in the domain of functions $\varphi(x)$ that satisfy

$$
\int_{0}^{\infty}|\varphi(x)|^{2} d x=\text { finite }
$$

and such that at the origin

$$
\frac{d \varphi\left(0^{+}\right)}{d x}=k \varphi\left(0^{+}\right)
$$

where $k$ is an arbitrary real constant[13]. As shown in reference [5] (see also the Appendix 2), this is the most general boundary condition confining a particle to the right side of the real line that makes the Hamiltonian self-adjoint. The physical significance of the boundary conditions is as follows: The infinite barrier implied by the usual boundary condition $\varphi\left(0^{+}\right)=0$ is neutral in the sense that it neither attracts nor repels the particle. In contrast, the infinite barrier implied by the boundary condition equation (9), although completely impenetrable, attracts the particle $(k<0)$ or repels $(k>0)$ it. It can be obtained from a combination of square well potentials using a limiting procedure [14] as shown in references [15], [16] and [17].

To see that the boundary condition (9) makes the Hamiltonian self-adjoint we have, integrating twice by parts, that

$$
\begin{aligned}
\int_{0}^{+\infty}(- & \left.\frac{\hbar^{2}}{2 m} \frac{d^{2}}{d x^{2}} \varphi_{1}(x)\right)^{*} \varphi_{2}(x) d x=\int_{0}^{+\infty} \varphi_{1}^{*}(x)\left(-\frac{\hbar^{2}}{2 m} \frac{d^{2}}{d x^{2}} \varphi_{2}(x)\right) d x \\
+ & \frac{\hbar^{2}}{2 m} \varphi_{2}^{*}(0) \varphi_{1}(0)\left[\frac{1}{\varphi_{1}(0)} \frac{d \varphi_{1}(0)}{d x}-\frac{1}{\varphi_{2}^{*}(0)} \frac{d \varphi_{2}^{*}(0)}{d x}\right] .
\end{aligned}
$$

Therefore only if both $\varphi_{1}(x)$ and $\varphi_{2}(x)$ satisfy the boundary condition equation (9), with the same constant $k$, we have $<\varphi_{1}\left|\left(\widehat{O} \varphi_{2}\right)>=<\left(\widehat{O}^{\dagger} \varphi_{1}\right)\right| \varphi_{2}>$ and the domain of the functions on the right (of the operator) is the same as the domain of the functions on the left (of the adjoint). Since the action of the operator and of its adjoint is the same, that is, $\widehat{O}=\widehat{O}^{\dagger}=-\frac{\hbar^{2}}{2 m} \frac{d^{2}}{d x^{2}}$, and the domain - determined by the boundary condition equation (9)- for both, the operator and its adjoint, is the same, the operator is self-adjoint. Note the delicate balance between the domain of the functions on the right (of the operator) and the domain of the functions on the left (of the adjoint): if we add, say, to the functions of the domain on the right-determined by the boundary condition (9)- functions such that $\varphi\left(0^{+}\right)$and $\frac{d \varphi\left(0^{+}\right)}{d x}=0$, we must add to the domain of the functions on the left -calculated with equation (3)- functions that, together with their derivatives, are different from zero at zero, with no relationship between them.( Examples of functions that have $\varphi\left(0^{+}\right)$and $\frac{d \varphi\left(0^{+}\right)}{d x}=0$ can be found in reference [5].)

Here we have a first example of how it is possible for textbooks to specify domains without mentioning it. Imposing boundary conditions specifies domains, and boundary conditions are imposed when the time-independent Schrödinger equation is solved. The boundary conditions are not arbitrary; they are imposed to make the operators self-adjoint in the domain they specify. In some cases, however, it is not necessary 
to impose boundary conditions to solve the time-independent Schrödinger equation. In Appendix 1 we give two examples of a Hamiltonian (the harmonic oscillator and a particle in moving in a line and subject to a finite square well potential) whose time-independent Schrödinger equation do not require specification of boundary condition on the wave functions to be self-adjoint. (In this case self-adjointness follows naturally from the action.)

\section{Example 1.5) The infinite square well potential}

Consider a particle moving in one dimension under the influence of an infinite square well, that is, $V(x)=0$ for $-L \leq x \leq L$ and $V(x)=\infty$ for $|x|>L$. It is natural to solve the problem by imposing the boundary condition $\Psi( \pm L)=0$. This boundary conditions makes the operator $H=-\frac{\hbar^{2}}{2 m} \frac{d^{2}}{d x^{2}}$ acting on the space of functions defined for $|x| \leq L$ such that $\int_{-L}^{L}|\Psi(x)|^{2} d x=$ finite self -adjoint. It is not, as the reader is probably suspecting, the most general boundary conditions. Indeed, given a two by two Hermitian matrix $M$, the following boundary conditions [18] makes $H=-\frac{\hbar^{2}}{2 m} \frac{d^{2}}{d x^{2}}$ self -adjoint

$$
\left[\begin{array}{l}
-\Psi^{\prime}(L) \\
\Psi^{\prime}(-L)
\end{array}\right]=M\left[\begin{array}{l}
\Psi(L) \\
\Psi(-L)
\end{array}\right] .
$$

The eigenvalues and eigenfunctions of this "generalized" infinite square well can be found in an article by Luz and Chen [19]. The physical significance of those more general boundary condition can be deduced from the example 1.4 when the matrix $M$ is diagonal. In the more general case the particle can disappear from $-L$ and reappear in $L$ or vice-versa. Therefore the system changes and the operator describes a particle moving in a closed path with an interaction in the now common point $(-L, L)$. (This kind of transmutation is common as is shown in other examples in reference [5].)

\section{Example 1.6) The hydrogen atom}

The wave function of the hydrogen atom after separation of variables reads $\Psi_{E l m}(r, \theta, \phi)=\frac{R_{E l}}{r} Y_{l m}(\theta, \phi)$. So the radial Hamiltonian of the hydrogen atom is given by

$$
\left\{\frac{d^{2}}{d r^{2}}+\frac{2 m}{\hbar^{2}}\left[E+\frac{e^{2}}{r}-\frac{l(l+1)}{2 m r^{2}}\right]\right\} R_{E l}(r)=0 .
$$

This operator acts on functions such that $\int_{0}^{\infty}\left(R_{E l}(r)\right)^{*} R_{E l}(r) d r=$ finite . It is usually argued that the boundary condition on $R_{E l}(r)$ should be $R_{E l}(0)=0$ so that the wave function $\Psi_{E l m}$ remains finite at $r=0$. Actually, we have the following situation: If $l>0$ ( that is $l=1,2, \ldots$ ) the condition $R_{E l}(0)=0$ specifies a domain such that the Hamiltonian given by equation (12) is self-adjoint and furthermore no other condition is possible. However, when $l=0$, although $R_{E 0}(0)=0$ makes the operator given by equation (12) self-adjoint, it is not the only possible choice. The following boundary condition is also admissible since it leads to a mathematically sound operator:

$$
\begin{aligned}
& \sin \alpha\left\{\lim _{r \rightarrow 0}\left[\frac{1}{2} R_{E l=0}(r)-r \frac{d R_{E l=0}(r)}{d r}\right]\right\} \\
& -\cos \alpha\left\{\lim _{r \rightarrow 0}\left[\frac{2}{\pi} R_{E l=0}(r) \log \left(\gamma^{\frac{1}{2}} r^{\frac{1}{2}}\right)-\frac{1}{\pi \gamma} \frac{d R_{E l=0}(r)}{d r}\right]\right\}=0,
\end{aligned}
$$

where $0 \leq \alpha<2 \pi$ is an arbitrary parameter and $\gamma=\frac{2 m}{\hbar^{2}} e^{2}$. In Appendix 2 we prove that the above boundary condition is admissible. It is also possible to demonstrate that the above boundary condition reduces to the one given by [20], page 53, equation 2.1.9. (It may be necessary to use the technique described in [5], Appendix B).

The physical significance of this boundary condition is that, in addition to the Coulomb interaction, we have an additional point interaction [20], with zero range, at the origin. At this point it is necessary to note that the modulus square of the wave function is a probability density, and so it can assume infinite values, at isolated points, if its integral, the probability, is finite. Therefore as long as $\int_{0}^{\infty}\left|\frac{R_{E 0}(r)}{r}\right|^{2} r^{2} d r$ is finite the fact that the wave function goes to infinite is not a problem [21].

\section{Example 1.7) Some exactly solvable problems}

The following problems from reference [22] can be solved by imposing to the s-wave radial function the boundary condition (9): The Wood -Saxon potential, page 162; The Hulthen potential, page 175; the Morse potential, page 182 . (In contrast, the Yukawa potential, page 189, can be solved by imposing boundary condition (13) to its s-wave radial function.)

In fact the radial Hamiltonian of those problems is

$$
\left\{\frac{d^{2}}{d r^{2}}+\frac{2 m}{\hbar^{2}}\left[E+V(r)-\frac{l(l+1)}{2 m r^{2}}\right]\right\} R_{E l}(r)=0
$$

where $V(r)$ is one of the above problems. For the first three potentials above, when $r \rightarrow 0$ the potentials go to zero . Hence using the approach given in the Appendix 2 we get the boundary condition (9) with $\varphi\left(0^{+}\right)$replaced by $R_{E 0}\left(0^{+}\right)$. The physical interpretation of this model is that at the origin we have a zero range interaction in this case the so called Fermi pseudopotential, $V_{F P P}=-\left(\frac{4 \pi}{k}\right) \delta(r) \frac{\partial}{\partial r}$, where $\delta(r)$ is the Dirac delta function. This pseudo potential have applications in atomic and condensed matter physics (see references [23] and [24]) and its equivalence to the boundary condition can be found in pages 46 to 49 of reference [20].

It should be mentioned that the point interaction behind the boundary condition (13) and the Fermi pseudopotentialbehind the boundary condition (9)- are too strong for a many body system. If we have, say, a three body system interacting through those interactions, although the system is self-adjoint, it has no finite energy ground state [25]. Therefore, to have a finite energy ground state is a property that should be required in addition to self-adjointness. The property of point interactions of having or not having a finite energy ground state in the case of a many body system is dimensional dependent: the many body system interacting through point interactions in one dimension is not 
only self-adjoint but also have a finite ground state energy [26]

Example 1.8) The one dimensional hydrogen atom

This example shows how the concept of self-adjointness can solve very tricky controversies. Consider the one dimensional hydrogen atom

$$
\frac{d^{2} \Psi}{d x^{2}}+\frac{2 m}{\hbar^{2}}\left[E+\frac{e^{2}}{|x|}\right] \Psi=0,-\infty<x<\infty .
$$

This problem was originally considered by Loudon [27]. His solution using $\Psi\left(0^{+}\right)=\Psi\left(0^{-}\right)=0$ is not the most general and considerable controversy about this problem was thus created. The complete solution-using the method given in Appendix 2- and a description of the controversy raised by Loudon's solution can be found in reference [28].

In the next section we discuss Stone's theorem and see why the Hamiltonian of a system must be self-adjoint for the timedependent Schrödinger equation to have a unique solution valid for all times.

\section{STONE'S THEOREM}

As mentioned in the introduction, self-adjointness of the Hamiltonian is equivalent to the unique solvability of the timedependent Schrödinger equation ([29], [30])

$$
i \hbar \frac{\partial \phi(x, t)}{\partial t}=H \phi(x, t)
$$

such that $\|\phi(x, t)\|^{2}=\|\phi(x, 0)\|^{2}$. To explain this result we first recall that there are two methods used in quantum mechanics textbooks to solve the time-dependent Schrödinger equation. The first one relies on the fact that a self-adjoint operator has a complete set of eigenfunctions. The eigenfuntions satisfy two properties: orthogonality and completeness. As a consequence, any "reasonable" function can be expanded as a series or integral (or a mixture of both) of those eigenfunctions. This result is used in many quantum mechanics textbooks. However, the fact that the eigenfunctions are sometimes distributions and that operators must be self-adjoint is never mentioned. The fact that very often one must restrict the domain of the operators in order to get a complete set is not mentioned either. Therefore we give below a few examples to clarify those issues.

\section{A. Orthogonality and completeness of the eigendistributions of a self-adjoint operator}

Example 2.1) The operator momentum

The operator momentum, $p=\frac{\hbar}{i} \frac{d}{d x}$, acting on the space given by equation (1), is self-adjoint in its "natural" domain so that there is no need to impose boundary conditions to restrict its domain. However, its eigenfunctions do not belong to the space given by equation (1), and hence cannot properly be called eigenfunctions. This is one of the reasons for the quotation mark encircling the word "eigenfuctions" used in the introduction, but a stronger reason is given in the next example. Those eigenfunctions are called eigenfunctions in the continuum and are given by $\psi_{\lambda}(x)=\frac{1}{(2 \pi)^{1 / 2}} e^{\frac{i}{\hbar} \lambda x}$. They satisfy orthogonality

$$
\int_{-\infty}^{\infty} \psi_{\lambda}^{*}(x) \psi_{\lambda^{\prime}}(x) d x=\int_{-\infty}^{\infty} \frac{1}{(2 \pi)^{1 / 2}} e^{\frac{i}{\hbar} \lambda x} \frac{1}{(2 \pi)^{1 / 2}} e^{\frac{i}{\hbar} \lambda^{\prime} x} d x=\delta\left(\lambda-\lambda^{\prime}\right)
$$

and completeness

$$
\int_{-\infty}^{\infty} \psi_{\lambda}^{*}(x) \psi_{\lambda}\left(x^{\prime}\right) d \lambda=\delta\left(x-x^{\prime}\right) .
$$

So that any function $\Psi(x)$ can be expanded as follows

$$
\Psi(x)=\int_{-\infty}^{\infty} F(\lambda) \frac{1}{(2 \pi)^{1 / 2}} e^{\frac{i}{\hbar} \lambda x} d \lambda ; \quad F(\lambda)=\int_{-\infty}^{\infty} \frac{1}{(2 \pi)^{1 / 2}} e^{-\frac{i}{\hbar} \lambda x} \Psi(x) d x .
$$

\section{Example 2.2) The operator $\hat{x}$}

Consider the operator $\hat{x}$ acting on the space of functions given by equation (1). As shown above, this operator is selfadjoint in its "natural " domain, so, again, there is no need to impose boundary conditions to restrict its domain. Its eigen- functions, however, are not functions, but distributions (also called generalized functions), in this case the Dirac delta function [31]. The eigenfunctions of $\hat{x}$ are

$$
\hat{x} \delta\left(x-x_{\lambda}\right)=x_{\lambda} \delta\left(x-x_{\lambda}\right)
$$


and they satisfy orthogonality

$$
\int_{-\infty}^{\infty} \delta^{*}\left(x-x_{\lambda}\right) \delta\left(x-x_{\lambda^{\prime}}\right) d x=\delta\left(x_{\lambda}-x_{\lambda^{\prime}}\right)
$$

and completeness

$$
\int_{-\infty}^{\infty} \delta^{*}\left(x-x_{\lambda}\right) \delta\left(x^{\prime}-x_{\lambda}\right) d x_{\lambda}=\delta\left(x-x^{\prime}\right) .
$$

Any function can be expanded as follows

$$
\Psi(x)=\int_{-\infty}^{\infty} \Psi\left(x_{\lambda}\right) \delta\left(x-x_{\lambda}\right) d x .
$$

The fact that self-adjoint operators have a complete set of "eigendistributions" is known as the Gelfand-Kostyuchenko theorem. This is not an easy theorem and a complete account of it can be found in a recent book by Zeidler [32].

\section{Example 2.3) Hamiltonians}

All the Hamiltonians, if they are self-adjoint, have a set of complete and orthogonal eigenfunctions. They are bound states eigenfunctions $\Psi_{E_{i}}$ or a set of continuum eigenfunctions $\Psi_{E}(x)$ or both, such that, we have orthogonality

$\int_{-\infty}^{\infty} \Psi_{E_{i}}^{*}(x) \Psi_{E_{j}}(x) d x=\delta_{E_{i} E_{j}} ; \int_{-\infty}^{\infty} \Psi_{E}^{*}(x) \Psi_{E^{\prime}}(x) d x=\delta\left(E-E^{\prime}\right)$,

where the symbol $\delta_{E_{i} E_{j}}$ is the Kroneker delta and $\delta\left(E-E^{\prime}\right)$ is the Dirac delta function - and completeness

$$
\sum_{E_{j}} \Psi_{E_{j}}^{*}(x) \Psi_{E_{j}}\left(x^{\prime}\right)+\int_{-\infty}^{\infty} \Psi_{E}^{*}(x) \Psi_{E}\left(x^{\prime}\right) d E=\delta\left(x-x^{\prime}\right) .
$$

Any function $\phi(x)$ can be expanded as

$$
\phi(x)=\sum c_{j} \Psi_{E_{i}}(x)+\int d E c(E) \Psi_{E}(x),
$$

where $c_{j}=\int_{-\infty}^{\infty} \Psi_{E_{i}}^{*}(x) \phi(x) d x$ and $c(E)=\int_{-\infty}^{\infty} \Psi_{E}^{*}(x) \phi(x) d x$. However, for Hamiltonians, it is usually necessary to restrict the domain by imposing boundary conditions, so that the operator becomes self-adjoint in this domain. For example, for the infinite square well of example (1.5), we have different sets of orthogonal and complete eigenfunctions depending on the parameters of the matrices $M$. This fact clearly illustrates the role of domains in getting a complete set of eigenfuntions: for each domain we have a different operator and hence we get a different set of eigenvalues and eigenfunctions

\section{B. Stone's theorem: first method}

Let's now return to the problem of solving equation (16). Once the eigenfunctions of the Hamiltonian are found out it is possible to expand the $t=0$ initial state $\phi(x, 0)$ in the eigenfunctions of the Hamiltonian:

$$
\phi(x, 0)=\sum c_{i} \Psi_{E_{i}}(x)+\int d E c(E) \Psi_{E}(x) .
$$

The solution of equation (16) is then given by

$$
\phi(x, t)=\sum c_{j} \Psi_{E_{i}}(x) e^{-\frac{i}{\hbar} E_{j} t}+\int d E c(E) \Psi_{E}(x) e^{-\frac{i}{\hbar} E t}
$$

as can be easily checked.

It is now possible to understand in a pedestrian way the connection between self-adjointness, domains, boundary conditions and the time-dependent Schrödinger equation. In fact when boundary conditions are imposed in the context of the time-independent Schrödinger equation, eigenvalues and eigenfunctions result. Those are assumed complete, as is correct since the Hamiltonian is self-adjoint in the domain imposed. Then expanding $\phi(x, 0)$ in this complete set the time dependent evolution can be derived. The connection between the domains and time evolution is full of consequences. For example, in the case of the finite square well, as we have seen above, for each set of parameters of the matrix $M$ (characterizing distinct operators since they have different domains) we have distinct sets of eigenvalues and eigenfunctions and therefore different time evolutions. However as we have seen above the eigenfunctions of a self-adjoint operator can be distributions. So it is nice to have a method of solution that do not depend on eigenfunctions expansions. We present this method below.

\section{Stone's theorem: second method}

The fact that the Hamiltonian must be self-adjoint is even more clearly seen in the second method to solve equation (16) that we will now present. This second method consists in constructing an operator $U(t)$ so that $\phi(x, t)=U(t) \phi(x, 0)$, with the following properties [30]:

a) $t \rightarrow U(t) \phi$ is continuous for each $\phi$ in the Hilbert space of the system

b) $U(t+s)=U(t) U(s)$ with $U(0)=1$

c) $\|U(t) \phi\|=\|\phi\|$ for all $t$ and for all $\phi$ in the Hilbert space of the system. Here $\|\phi\|$ means the norm of $\phi$, defined above in equation (1), and the property c) ensures conservation of probability. The family of operators $U(t)$ is called a unitary one-parameter group. An alternative definition of self-adjointness is that an operator $H$ is self-adjoint if and only if there is a unitary one-parameter group family of operators, $U(t)$, so that $t \rightarrow U(t)$ is differentiable with $\frac{\partial U(t) \phi}{\partial t}=-i H U(t) \phi$. (Note that intuitively, that is, formally differentiating with respect to $t, \exp \left[-\frac{i}{\hbar} H t\right] \varphi(x, 0)$ is the solution of equation (16))

The equivalence of this definition of self-adjointness with the more usual (given in section 2), is the content of Stone's theorem [29]. To qualitatively understand this theorem in terms of domains it is necessary to construct the operator $U(t)$ given by $\exp \left[-\frac{i}{\hbar} H t\right]$. In fact to be sure that $\exp \left[-\frac{i}{\hbar} H t\right]$ makes sense, we have to show how to construct it from $H$, and this is the objective of the next section. (In passing we mention that the existence of the operator $\exp \left[-\frac{i}{\hbar} H t\right]$ is also essential to transform from the Schrödinger picture to the Heisenberg picture and vice-versa as emphasized by Dirac [33].) 


\section{CONSTRUCTING THE OPERATOR $\exp \left[-\frac{i}{\hbar} H t\right]$}

In this section we follow reference [30] closely and, in particular, we use units such that $\hbar=1$, to simplify the notation. A natural way of making sense of the operator exponential is to use the compound interest formula [34],

$$
e^{-i H t}=\lim _{n \rightarrow \infty}\left(1+\frac{i H t}{n}\right)^{-n}
$$

It is necessary to find out what are the conditions that $H$ must satisfy so that this limit exists. For this we rewrite equation (28) distinguishing positive $t$ from negative $t, t= \pm|t|$ as

$$
\begin{aligned}
e^{-i H t} & =\lim _{n \rightarrow \infty}\left(1 \pm \frac{i|t| H}{n}\right)^{-n}=\lim _{n \rightarrow \infty}( \pm i)^{-n}\left(\frac{|t| H}{n} \mp i\right)^{-n} \\
& =\lim _{n \rightarrow \infty}( \pm i)^{-n}(\alpha H \mp i)^{-n}=\lim _{n \rightarrow \infty}( \pm i)^{-n} A^{n}
\end{aligned}
$$

where $\alpha=\frac{|t|}{n}$ and $A=(\alpha H \mp i)^{-1}$.

To deduce the conditions for equation (29) to make sense we note that for any finite $n$ equation (29) is a product of operators $A=(\alpha H \pm i)^{-1}$. We begin to investigate the existence of this operator by examining its inverse, the operator $(\alpha H \pm i)$. By looking at what are the functions $\psi(x)=(\alpha H \pm i) \varphi(x)$ where $\varphi(x)$ belongs to the domain of $H$, we can examine the range of $(\alpha H \pm i)$, that is the set of all the functions $\Psi(x)$ that results from applying $(\alpha H \pm i)$ to all the functions in its domain. Calculating the norm of $\psi(x)$ we have,

$$
\begin{gathered}
<\psi|\psi>=<(\alpha H \pm i) \varphi|(\alpha H \pm i) \varphi>=\alpha^{2}<H \varphi \mid(H \varphi> \\
\mp i \alpha<\varphi|H \varphi> \pm i \alpha<H \varphi| \varphi>+( \pm i)(\mp i)<\varphi \mid \varphi>.
\end{gathered}
$$

At this stage there is no need for $H$ to be defined in a domain such that it is self-adjoint; it suffices to be Hermitian for the last two terms to cancel out. The first term can be written $\alpha^{2}<H \varphi \mid\left(H \varphi>=\alpha^{2}<\varphi \mid\left(H^{2} \varphi\right)>\geq 0\right.$ and so we have

$$
\|(\alpha H \pm i) \varphi\|^{2}=\alpha^{2}<\varphi \mid\left(H^{2} \varphi\right)>+\|\varphi\|^{2} \geq\|\varphi\|^{2} .
$$

Therefore we can define an operator $A=(\alpha H \pm i)^{-1}$ from the range of $(\alpha H \pm i)$, to the domain of $H$. Let $\psi(x)$ be one such function. It clearly obeys

$$
\|A \psi\|=\left\|(\alpha H \pm i)^{-1} \psi\right\| \leq\|\psi\| .
$$

Indeed let $\varphi(x)$ belong to the domain of $H$ and let $\psi(x)$ belong to the range of $(\alpha H \pm i)$ such that

$$
(\alpha H \pm i) \varphi(x)=\psi(x) .
$$

Of course we have

$$
A \psi(x)=(\alpha H \pm i)^{-1} \psi(x)=\varphi(x)
$$

and replacing equation (34) in equation (31) we have

$$
\|\psi\|^{2} \geq\|A \psi\|^{2}
$$

from which it is possible to conclude that in general the operator $A$ contracts the function on which it acts. So, unless the range of $(\alpha H \pm i)$ is the whole Hilbert space, repeated application of $A$ as implied by equation (29) results in smaller and smaller sets of functions. So we are forced to demand that the domain of $A$ be the whole Hilbert space. It turns out that if $H$ is self-adjoint the domain of $A$, which is the range of $(\alpha H \pm i)$, is the entire Hilbert space. This is a fundamental theorem: $H$ is self-adjoint if and only if the range of $(\alpha H \pm i)(\alpha>0)$ is the entire Hilbert space.

We will not demonstrate this theorem here but give an example (for the operator $\hat{x}$ and $\alpha=1$ ) that takes a little bit of the mystery of this result and refer to Amrein [35] and to Reed and Simon [36] for the complete demonstration. We know that if $\varphi(x)$ is a function belonging to the domain of $\hat{x}$ then $\hat{x} \varphi(x)=x \varphi(x)$ also belongs to the Hilbert space, that is it obeys equation (4). However, another application of $\hat{x}$ may result in a function outside the Hilbert space, that is $\int_{-\infty}^{+\infty}\left|x^{2} \varphi(x)\right|^{2} d x=\infty$. It is easy to understand that $(x \pm i) \varphi(x)$ belongs to the Hilbert space, if $x \varphi(x)$ does. However how do we know that the range of $(x \pm i)$ is the entire Hilbert space? We want to show that if a function $h(x)$ is orthogonal to a function $\psi$ in the range of $(\hat{x} \pm i)$ - that is $\psi=(\hat{x} \pm i) \varphi$ where $\varphi$ belongs to the domain of $x$ - then $h(x)=0$. In fact if $h$ is orthogonal to the range of $(\hat{x} \pm i)$ then we have $\hat{x} h \pm i h=0$ (because, $(h,(\hat{x} \pm i) \varphi)=0$, and $(h,(\hat{x} \pm i) \varphi)=((\hat{x} \mp i) h, \varphi)=0)$ and therefore, by the criterion given in example $1.8, h(x)=0$. It is not difficult to extend this demonstration to any $H$. However, although it is a fact that the range of $(\alpha H \pm i)$ is the entire Hilbert space this does not yet show that repeated application of $(\alpha H \pm i)^{-1}$ converges to a limit. A complete demonstration, for which self-adjointness is essential, can be found in the book by Kato [37]

\section{v. CONCLUSIONS}

In this paper we have shown that the seemingly complicated and unnecessary requirement of self-adjointness of the Hamiltonians in quantum mechanics is actually essential to define the exponential of such operators and therefore solve the time-dependent Schrödinger equation uniquely. This point is largely overlooked in textbooks in quantum mechanics. This is probably because the definition of selfadjointness requires that operators be correctly defined by their action and by their domains. We have shown that domains are specified when boundary conditions are imposed at the stage of solving the time-independent Schrödinger equation. However, in textbooks, the specification of boundary conditions is not presented as a domain specification that turns the Hamiltonian self-adjoint. The boundary conditions are usually justified with physical arguments or simply glossed over. We find this unsatisfactory and think that it is necessary to be aware of the importance of domains, and of its relation with self-adjointness, which is essential for the timedependent Schrödinger equation to have a unique-probability conserving-solution for all times. 


\section{Appendix 1: Examples of a Hamiltonians that does not require boundary conditions for the specification of its domain}

In this Appendix we give two examples of systems whose Hamiltonians are self-adjoint, without the need of boundary condition in their wave functions to have their domains specified.

Example 1) The time-independent Schrödinger equation for the harmonic oscillator is

$$
\frac{-\hbar^{2}}{2 m} \frac{d^{2} \Psi(x)}{d^{2} x^{2}}+\frac{1}{2} k x^{2} \Psi(x)=E \Psi(x) .
$$

We want to show that the equations

$$
\frac{-\hbar^{2}}{2 m} \frac{d^{2} \Psi_{ \pm}(x)}{d^{2} x^{2}}+\frac{1}{2} k x^{2} \Psi(x)= \pm i \eta \Psi(x)
$$

has no square integrable solution. The usual way to tackle equation (36) is to apply Frobenius method directly to the equation. This, however, is not without its problems, as shown in references [38] and [39] and has lead to errors in the pedagogical literature [40], corrected in references [41] and [42]. Therefore, we prefer to transform (37) as done in reference [43], so that we can use the powerful methods described in Olver's book [44].

First set $k=\omega^{2} m$, change variables $\xi=\left(\frac{m \omega}{\hbar}\right)^{1 / 2} x$ and define $v_{ \pm}(\xi)=\Psi_{ \pm}(x)$ to get

$$
\frac{d^{2} v_{ \pm}(\xi)}{d \xi^{2}}+\left( \pm \frac{2 i \eta}{\hbar \omega}-\xi^{2}\right)^{2} v_{ \pm}(\xi)=0
$$

Next set

$$
v_{ \pm}(\xi)=e^{-\frac{\xi^{2}}{2}} f_{ \pm}(\xi)
$$

to get

$$
\frac{d^{2} f_{ \pm}(\xi)}{d \xi^{2}}-2 \xi \frac{d f_{ \pm}(\xi)}{d \xi}+2 \mu_{ \pm} f_{ \pm}(\xi)=0
$$

where $\mu_{ \pm}=-\frac{1}{2} \pm \frac{i \eta}{\hbar \omega}$. Finally, a further change $z=\xi^{2}$ and $F_{ \pm}(z)=f_{ \pm}(\xi)$ gives

$$
z \frac{d^{2} F_{ \pm}(z)}{d z^{2}}+\left(\frac{1}{2}-z\right) F_{ \pm}(z)+\frac{\mu_{ \pm}}{2} F_{ \pm}(z)=0
$$

Equation (41) is the confluent hypergeometric equation, which has the following two independent solutions [45]

$$
{ }_{1} F_{1}\left(-\frac{\mu_{ \pm}}{2}, \frac{1}{2} ; z\right) \quad \text { and } \quad z_{1}^{\frac{1}{2}} F_{1}\left(\frac{1}{2}-\frac{\mu_{ \pm}}{2}, \frac{3}{2} ; z\right) \text {. }
$$

An arbitrary combination of these functions behaves as $e^{z}$ for large $z$. However, the two combinations below are such that one of them,$\Psi_{+}(x)$, goes to infinite as $e^{x^{2}}$ as $z \rightarrow-\infty$ and to $x^{\mu_{ \pm}}$as $z \rightarrow \infty$ and the other,$\Psi_{-}(x)$, that goes as $e^{x^{2}}$ as $z \rightarrow \infty$ and to $x^{\mu \pm}$ as $x \rightarrow-\infty$.

$$
\begin{aligned}
& \Psi_{+}(x)=\frac{\Gamma\left(\frac{1}{2}\right){ }_{1} F_{1}\left(-\frac{\mu_{ \pm}}{2}, \frac{1}{2} ;\left(\frac{m \omega}{\hbar}\right) x^{2}\right)}{\Gamma\left(\frac{1}{2}-\frac{\mu}{2}\right)}+\frac{\Gamma\left(-\frac{1}{2}\right)\left(\frac{m \omega}{\hbar}\right)^{\frac{1}{2}} x_{1} F_{1}\left(\frac{1}{2}-\frac{\mu_{ \pm}}{2}, \frac{3}{2} ;\left(\frac{m \omega}{\hbar}\right) x^{2}\right)}{\Gamma\left(-\frac{\mu}{2}\right)}, \\
& \Psi_{-}(x)=\frac{\Gamma\left(\frac{1}{2}\right){ }_{1} F_{1}\left(-\frac{\mu_{ \pm}}{2}, \frac{1}{2} ;\left(\frac{m \omega}{\hbar}\right) x^{2}\right)}{\Gamma\left(\frac{1}{2}-\frac{\mu \pm}{2}\right)}-\frac{\Gamma\left(-\frac{1}{2}\right)\left(\frac{m \omega}{\hbar}\right)^{\frac{1}{2}} x_{1} F_{1}\left(\frac{1}{2}-\frac{\mu_{ \pm}}{2}, \frac{3}{2} ;\left(\frac{m \omega}{\hbar}\right) x^{2}\right)}{\Gamma\left(-\frac{\mu \pm}{2}\right)} .
\end{aligned}
$$

For real $\mu_{ \pm}$such solutions can be matched together with their derivatives at $x=0$ and, as discussed nicely in reference [43], results in the Legendre polynomials. However if $\mu_{ \pm}$ is imaginary they cannot be matched smoothly at $z=0$ and hence equation (37) has no solutions. So according to the criterion given by equation (6) the operator is self-adjoint.

Example 2-Consider a particle moving in one dimension and subject to the finite square well potential , that is $V(x)=$ $-V_{0}$ for $|x|<L$ and $V(x)=0$ for $|x| \geq L$. This system, whose Hamiltonian is $H=-\frac{\hbar^{2}}{2 m} \frac{d^{2}}{d x^{2}}+V(x)$, is self-adjoint in its "natural" domain. The boundary condition that must be imposed at $\pm L$ assures that the functions in the domain $\varphi(x)$ remain in the domain when acted with the term $-\frac{\hbar^{2}}{2 m} \frac{d^{2}}{d x^{2}}$, which is part of the Hamiltonian.

\section{Appendix 2: The boundary conditions at $r=0$ of the Hydrogen Atom}

A recipe to get the boundary conditions can be found in [5]. However in this case it is simpler to follow a prescription given by Krall [46]. It consists in the following steps

1) Solve the differential equation

$$
\left[\frac{d^{2}}{d r^{2}}+\frac{\gamma}{r}\right] \Psi^{ \pm}(r)=0
$$


obtained from equation (12) by making $l=0, \gamma=\frac{2 m}{\hbar^{2}} e^{2}$, and $E=0$. This equation can be obtained from the equation below (See Abramowitz, [47], equation 9.1.50)

$$
\frac{d^{2} \Psi}{d r^{2}}+\left(\frac{\lambda^{2}}{4 r}-\frac{v^{2}-1}{4 r^{2}}\right) \Psi=0
$$

making $v=1$ and $\lambda=2 \gamma^{\frac{1}{2}}$, and whose solutions are $\Psi(r)=$ $r^{\frac{1}{2}} C_{v}\left(\lambda r^{\frac{1}{2}}\right)$, where $C_{v}(z)$ is any of the Bessel's Functions. The two linearly independent solutions of equation (45) are therefore

$$
\Psi_{1}=r^{\frac{1}{2}} J_{1}\left(\lambda r^{\frac{1}{2}}\right)
$$

and

$$
\Psi_{2}=r^{\frac{1}{2}} N_{1}\left(\lambda r^{\frac{1}{2}}\right)
$$

where $J_{1}(z)$ and $N_{1}(z)$ are the Bessel and Newman functions, respectively.

2) The second step is to compute

$$
\lim _{r \rightarrow 0}\left\{\sin \alpha W\left(\varphi, \Psi_{1}\right)+\cos \alpha W\left(\varphi, \Psi_{2}\right)\right\}=0
$$

where $0 \leq \theta \leq 2 \pi$ is a real number and $W\left(f_{1}, f_{2}\right)$ is the Wronskian of $f_{1}$ and $f_{2}$. This gives us a relation between $\lim _{r \rightarrow 0} R_{E l=0}(r)$ and $\lim _{r \rightarrow 0} \frac{d R_{E l=0}}{d r}$ which is the boundary condition

The same procedure can be applied to the operator $-\frac{\hbar^{2}}{2 m} \frac{d^{2}}{d x^{2}}$ acting on functions on the space of functions that obeys $\int_{0}^{+\infty}|\varphi(x)|^{2} d x=$ finite (and that of course remains in the space) and results in the boundary condition given by equation (9).

\section{Acknowledgements}

This work was supported the Conselho Nacional de Desenvolvimento Científico e Tecnológico ( $\mathrm{CNPq})$, by the Fundação de Amparo a Pesquisa do Estado de São Paulo (Fapesp) and by the Japan Society for the Promotion of Sciences. The authors would like to thank prof. Y. Nogami very much for his help.
[1] Eugen Merzbacher, Quantum mechanics, third edition (John Wiley and Sons, inc. New York, 1988).

[2] M. H. Stone, Ann. Math. 33, 643 (1932).

[3] Consider an operator $\widehat{A}$. Only for self-adjoint operators we can define the operator $\exp \left[-\frac{i}{\hbar} \widehat{A} l\right]$ where $l$ is a real parameter. The ability to define the exponential of an operator is essential in studying the symmetry of the system. For example if $\widehat{A}$ is taken to be the operator momentum in the $x$ direction, $p_{x}=\frac{\hbar}{i} \frac{d}{d x}$, then the operator $\exp \left[-\frac{i}{\hbar} l p_{x}\right]$ represents a finite translation of the system in the $x$ direction by a distance $l$.

[4] G. Bonneau, J. Faraut, and G. Valent, Am. J. Phys. 69, 322 (2001).

[5] V. S. Araujo, F.A.B. Coutinho, and J.F. Perez, Am. J. Phys 72, 203 (2004)

[6] P. Garbaczewski and W. Karwowski, Am. J. Phys. 72, 924 (2004).

[7] A. M. Essin and D. J. Griffiths, Am. J. Phys. 74, 109 (2006).

[8] T. F. Jordan, Am. J. Phys. 44, 567 (1976).

[9] A. Z. Capri, Am. J. Phys. 45, 823 (1977).

[10] C. Zhu and J. R. Klauder, Am. J. Phys. 61, 605 (1993).

[11] F. Gieres, Rep. Prog. Phys. 63, 1893 (2000).

[12] The Dirac delta function is not a function in the sense that we can not associate a numerical value to it for each $x$. Occasionally we find $\delta\left(x-x_{\lambda}\right)=0$ if $x \neq x_{\lambda}$ and $\delta\left(x-x_{\lambda}\right)=\infty$ if $x=x_{\lambda}$. However multiplying this by, say, 2 we find $2=1$ ! In other occasions we find $\delta\left(x-x_{\lambda}\right)=0$ if $x \neq x_{\lambda}$ and $\delta\left(x-x_{\lambda}\right)=$ undefined if $x=x_{\lambda}$. However in this case one can not define $\left(\delta\left(x-x_{\lambda}\right)\right)^{2}=$ $\delta\left(x-x_{\lambda}\right) \delta\left(x-x_{\lambda}\right)$ for integrating this we find $\delta(0)$ which we declared undefined.

[13] Since we are going to integrate by parts we have to restrict the set of functions. So we take as the domain the set of functions that obey equation (8), that are absolutely continuous, and that go to zero as $x \rightarrow \infty$. See reference [5] for details.

[14] Note that the usual condition for an impenetrable, $U(0)=0$ is contained in equation (9) by making $k \rightarrow \infty$. This boundary condition is considered more natural because of the following limiting procedure. One considers the potential $V(x)=V_{0}>0$ for $x<0$ and $V(x)=0$ for $x>0$, solves the time- independent Schrödinger equation for an energy $E<V_{0}$ and then takes the limit $V_{0} \rightarrow \infty$. By doing this one gets the condition $U(0)=0$. However one can consider the following potential: $V(x)=V_{0}$ for $x<0, V(x)=B$, for $0<x<\varepsilon$ and $V(x)=0$ for $x>\varepsilon$. Solve the time- independent Schrödinger equation and find the scattering length. Then one takes $V_{0} \rightarrow \infty$. Now it is possible to let $\varepsilon \rightarrow 0$ together with $B \rightarrow-\infty$ in such away that the scattering length remains constant. In this way one gets equation (9) with $k<0(B \rightarrow-\infty)$; the scattering length is positive and there is a bound state. Alternatively letting $\varepsilon \rightarrow 0$ together with $B \rightarrow \infty$ we get equation (9) with $k>0$ in which case the scattering length is negative and there is no bound state.

[15] J. F. Perez and F. A. B. Coutinho, Am. J. Phys. 59, 52 (1991).

[16] F. A. B. Coutinho, Y. Nogami, and J. F. Perez, Phys. Rev. A 46, 6052 (1992).

[17] F. A. B. Coutinho, Y. Nogami, J. F. Perez, and M. F. Toyama, Journal of Physics A - Mathematical and General 27, 6539 (1994).

[18] M. Carreau, E. Farhi, and S. Gutmann, Phys. Rev. D 42, 1194 (1990).

[19] M. G. E. da Luz and B. K. Cheng, Phys. Rev. A 51, 1811 (1995).

[20] S. Albeverio , F. Gesztezy, R. Hoegh-Krogn, and H. Holden, Solvable models in Quantum Mechanics (Springer -Verlag, New York, 1988), page 54.

[21] Most books present arguments for disregarding the possibility of wave functions that are infinite at a point, but that has the probability finite; reference [1] on page 264 , correctly asserts, that the usual boundary condition $R_{E 0}(0)=0$ is imposed to make the Hamiltonian self-adjoint, and that other conditions are possible. (However, the exercise 12.8 of this reference is incorrect.) Among the many incorrect arguments given to sup- 
port the boundary condition $R_{E} 0(0)=0$, reference [22], (on page 157 of the volume 1) presents the following interesting argument: If the wave function at $r=0$ is allowed to behave as $\Psi_{E l=0 m=0}(r, \theta, \phi)=\frac{R_{E l}}{r} \sim \frac{1}{r}$, then the kinetic energy $\int_{0}^{\infty} \Psi_{E, l=0, m=0}^{*}(r)\left(-\frac{\hbar^{2}}{2 m} \nabla^{2} \Psi_{E, l=0, m=0}(r)\right) r^{2} d r$ diverges. However if we consider the entire Hamiltonian, $\int_{0}^{\infty} \Psi_{E, l=0, m=0}^{*}(r)\left(-\frac{\hbar^{2}}{2 m} \nabla^{2}+\right.$ $\left.V(r))^{2} \Psi_{E, l=0, m=0}(r)\right) r^{2} d r$, where the wave function obeys the boundary condition given by equation (13), the divergence in the kinetic energy is exactly cancelled by the divergence in the potential energy. This is a nice example of an operator that is formally a sum of two operators but whose domain is not the intersection of the natural domains of these operators taken individually.

[22] S. Flugge, Practical Quantum Mechanics, Volume 1 (SpringerVelag, New York, 1970).

[23] M. L. Cohen, Am. J. Phys. 52, 695 (1984).

[24] M. L. Cohen,"Looking back and ahead at condensed matter physics," Physics Today June 2006 pp 48-53.

[25] G. Flamand, "Mathematical theory of non-relativistic two-and three -particle systems with point interactions," in Cargese Lectures in Theoretical Physics 1967 edited by F. Lurçat.

[26] F. A. B. Coutinho, Y. Nogami Y, and L. Tomio, "Many-body system with a four-parameter family of point interactions in one dimension," Journal of Physics A-Mathematical and General 32, 4931 (1999).

[27] R. J. Loundon, Am. J. Phys. 27, 649 (1959).

[28] I. Tsutsui, T. Fülöp, and T. Cheon, J. Phys. A: Math. Gen. 36, 275 (2003)

[29] B. Simon, Acta Physica Austriaca, Suppl XVII, 19 (1997).

[30] Michael Reed and Barry Simon, Functional Analysis (Academic Press, San Diego 1980) Vol. 1, page 264.

[31] The Dirac delta function is in fact a functional that associates to each function a number. In this sense the eigenfunctions of $\hat{p}, \psi_{\lambda}(x)=\frac{1}{(2 \pi)^{1 / 2}} e^{\frac{i}{\hbar} \lambda x}$-or of any Hamiltonian-can also be considered a functional: $F(\lambda)=\int_{-\infty}^{\infty} \frac{1}{(2 \pi)^{1 / 2}} e^{-\frac{i}{\hbar} \lambda x} \Psi(x)$. In this case we call the resulting distribution a regular distribution in comparison with the delta function which is an irregular distribution, that is, not generated by a function. So we can announce a more general theorem than the one taken from granted in most quantum mechanics textbooks, the Gelfand-
Kostyuchenko theorem: Any self-adjoint operator have a complete set of eigendistributions[32]

[32] E. Zeidler, Quantum Field Theory I: Basics in Mathematics and Physics (Springer, Berlin 2006 ) page 679.

[33] P. A. M. Dirac, Lectures in Quantum Field theory, Belfer Graduate school of Science. Yeshiva University. New York (1966).

[34] At this point one may feel that expanding the exponential in power series would give us a better chance to define the exponential operator. It is indeed natural at this stage to think that using the series development of the operator $\exp \left[-\frac{i}{\hbar} H t\right]$ $=1-\frac{i}{\hbar} H t+.$. is a better strategy to define the exponential. However the series only converges when acting on analytic functions. See B. S. Holstein and A. R. Swift "Spreading wave packets-A cautionary Note.”Am. J. Phys. 40: 829-832 (1972) for a striking example.

[35] W. O. Amrein, Non-relativistic Quantum Dynamics (D.Reidel Publishing Company, Dordrecht, 1981) p. 29.

[36] M. Reed and B. Simon, Methods of Modern Mathematical Physics-Vol 2-Fourier Analysis, Self-Adjontness (Academic Press, NY, 1975), theorem X.1.

[37] T. Kato, Pertubation Theory for Linear Operators (SpringerVerlag Berlin 1980), p. 481.

[38] H. A. Buchdahl, Am. J. Phys. 42, 47 (1974).

[39] M. Bowen and J. Coster, Am. J. Phys. 48, 307 (1980).

[40] R. S. Bhalerao and B. Ram, Am. J. Phys. 68, 817 (2001).

[41] A. S. de Castro, "Comment on "Fun and frustration with the quarkonium in a $1+1$ dimension, [Am. J. Phys. 68, 817 (2001)]," Am. J. Phys.70, 450 (2002)

[42] R. M. Cavalcanti, "Comment on "Fun and frustration with quarkonium in a $1+1$ dimension," by R. S. Bhalerao and B. Ram, [Am. J. Phys. 69, 817 (2001)]," Am. J. Phys. 70, 451 (2002).

[43] W. Edward Gettys and H. W. Graben, Am. J. Phys. 43, 626 (1975).

[44] Frank W. J. Olver, Asymptotics and special functions (AKP Classics. AK Peters Wellesley, Massachusetts, 1997)

[45] A. Ederly, Higher transcendental functions (McGraw -Hill, New York, 1973).

[46] A. M. Krall, Journal of Differential equations 45, 128 (1982).

[47] M. Abramowitz and I. A. Stegun Handbook of Mathematical functions (Dover Publications, Inc., New York, 1965). 